\title{
On interpolation of the measure of noncompactness
}

\author{
Fernando Cobos $^{\mathrm{a}}$, Luz M. Fernández-Cabrera ${ }^{\mathrm{b}}$, and Antón Martínez ${ }^{\mathrm{c}}$ \\ a Departamento de Análisis Matemático, Facultad de Matemáticas, Universidad \\ Complutense de Madrid, 28040 Madrid, Spain; cobos@mat.ucm.es \\ b Sección Departamental de Matemática Aplicada, Escuela de Estadística, Universidad \\ Complutense de Madrid, 28040 Madrid, Spain; luz_fernandez-c@ mat.ucm.es \\ c Departamento de Matemática Aplicada I, E.T.S. Ingenieros Industriales, Universidad de \\ Vigo, 36200 Vigo, Spain; antonmar@uvigo.es
}

Received 10 April 2006

Abstract. We revised the known results on interpolation of the measure of noncompactness and we announce a new approach to establishing the interpolation formula for the real method.

Key words: real interpolation, measure of noncompactness.

\section{INTRODUCTION AND PRELIMINARIES}

Let $\bar{A}=\left(A_{0}, A_{1}\right)$ be a Banach couple, i.e., two Banach spaces $A_{j}, j=0,1$, which are continuously embedded in a common Hausdorff topological vector space, and let

$$
K(t, a)=\inf \left\{\left\|a_{0}\right\|_{A_{0}}+t\left\|a_{1}\right\|_{A_{1}}: a=a_{0}+a_{1}, a_{j} \in A_{j}\right\}, \quad a \in A_{0}+A_{1},
$$

and

$$
J(t, a)=\max \left\{\|a\|_{A_{0}}, t\|a\|_{A_{1}}\right\}, \quad a \in A_{0} \cap A_{1},
$$

be Peetre's $K$ - and $J$-functionals. As it is well known, for $0<\theta<1$ and $1 \leq q \leq \infty$, the real interpolation space $\left(A_{0}, A_{1}\right)_{\theta, q}$, realized in a discrete way as a $K$-space, is formed by all those $a \in A_{0}+A_{1}$ which have a finite norm

$$
\|a\|_{\theta, q ; K}=\left(\sum_{m=-\infty}^{\infty}\left(2^{-\theta m} K\left(2^{m}, a\right)\right)^{q}\right)^{1 / q}
$$


(the sum should be replaced by the supremum if $q=\infty$ ). We denote by $\bar{A}_{\theta, q ; K}$ the space $\left(A_{0}, A_{1}\right)_{\theta, q}$ normed by $\|\cdot\|_{\theta, q ; K}$. This space can be defined equivalently by using the $J$-functional. It turns out that $a$ belongs to $\left(A_{0}, A_{1}\right)_{\theta, q}$ if it can be written as a sum $a=\sum_{m=-\infty}^{\infty} u_{m}$ (convergence in $A_{0}+A_{1}$ ), where $\left\{u_{m}\right\} \subseteq A_{0} \cap A_{1}$ and

$$
\left(\sum_{m=-\infty}^{\infty}\left(2^{-\theta m} J\left(2^{m}, u_{m}\right)\right)^{q}\right)^{1 / q}<\infty .
$$

Moreover, the infimum of all values (1.1) over all possible representations of $a$ as above defines a norm $\|a\|_{\theta, q ; J}$ which is equivalent to $\|\cdot\|_{\theta, q ; K}$. We write $\bar{A}_{\theta, q ; J}$ for the space $\left(A_{0}, A_{1}\right)_{\theta, q}$ normed by $\|\cdot\|_{\theta, q ; J}$. By $\bar{A}_{\theta, q}=\left(A_{0}, A_{1}\right)_{\theta, q}$ we mean any of the spaces $\bar{A}_{\theta, q ; K}$ or $\bar{A}_{\theta, q ; J}$. Full details on the real method can be found in $\left.{ }^{1,2}\right]$.

Let $\bar{B}=\left(B_{0}, B_{1}\right)$ be another Banach couple, and let $T \in \mathcal{L}(\bar{A}, \bar{B})$, that is, $T$ is a linear operator from $A_{0}+A_{1}$ into $B_{0}+B_{1}$ whose restriction to each $A_{j}$ defines a bounded operator from $A_{j}$ into $B_{j}$ for $j=0,1$. It is well known that the restriction $T:\left(A_{0}, A_{1}\right)_{\theta, q} \longrightarrow\left(B_{0}, B_{1}\right)_{\theta, q}$ is bounded and the following inequality holds:

$$
\|T\|_{\bar{A}_{\theta ; q}, \bar{B}_{\theta ; q}} \leq C\|T\|_{A_{0}, B_{0}}^{1-\theta}\|T\|_{A_{1}, B_{1}}^{\theta} .
$$

Similar formulae have been established for other quantities (see $\left[{ }^{3-5}\right]$ ), but now some of them require much effort to be proved. This is the case of the measure of noncompactness, a concept that means more than only continuity but not so much as compactness.

Given a bounded linear operator $T \in \mathcal{L}(A, B)$, between the Banach spaces $A$ and $B$, we recall that the measure of noncompactness $\beta(T)=\beta\left(T_{A, B}\right)$ of $T$ is defined as the infimum of all $\sigma>0$ such that there exists a finite subset $\left\{b_{1}, \ldots, b_{s}\right\} \subseteq B$ so that

$$
T\left(U_{A}\right) \subseteq \bigcup_{j=1}^{s}\left\{b_{j}+\sigma U_{B}\right\} .
$$

Here, $U_{A}$ stands for the closed unit ball of $A$. The symbol $U_{B}$ has a similar meaning. It is clear that $\beta\left(T_{A, B}\right) \leq\|T\|_{A, B}$, and $T$ is compact if and only if $\beta(T)=0$ (see $\left[{ }^{6}\right]$ for more details on this notion).

In 1999, Fernández-Martínez and two of the present authors established the following interpolation formula for the measure of noncompactness:

$$
\beta\left(T_{\bar{A}_{\theta ; q}, \bar{B}_{\theta ; q}}\right) \leq C \beta\left(T_{A_{0}, B_{0}}\right)^{1-\theta} \beta\left(T_{A_{1}, B_{1}}\right)^{\theta} .
$$

The proof is based on the vector-valued sequence spaces that come up when defining the real interpolation space. Using them and certain families of projections, one can decompose and approximate the operator $T$ by other operators whose measure of noncompactness can be directly estimated. This technique has 
its origin in the papers $\left[{ }^{7-10}\right]$. Previous results on interpolation of the measure of noncompactness are due to Edmunds and Teixeira $\left[{ }^{11}\right]$. The results of $\left[{ }^{11}\right]$ refer to the special case when $A_{0}=A_{1}$, or $B_{0}=B_{1}$, or the couple $\left(B_{0}, B_{1}\right)$ satisfies a certain approximation condition. Under these extra assumptions, the proofs are easier and results also apply to the complex method. However, it is an open problem if a similar formula to (1.2) holds for the complex method.

The aim of this note is to announce a new approach to (1.2), which simplifies the way of handling the "middle parts" of the operator. The main lines of the proof are described in the next section. Full details can be found in the paper $\left[{ }^{12}\right]$.

\section{SKETCH OF THE MAIN LINES OF THE PROOF}

In order to establish (1.2), we realize $\bar{A}_{\theta, q}$ as a $J$-space and $\bar{B}_{\theta, q}$ as a $K$-space. This will allow us to work with the vector-valued sequence spaces which are implicit in those descriptions. Namely, for $m \in \mathbb{Z}$, we denote by $G_{m}$ the Banach space $A_{0} \bigcap A_{1}$ endowed with the norm $J\left(2^{m}, \cdot\right)$, and by $F_{m}$ we mean $B_{0}+B_{1}$ normed by $K\left(2^{m}, \cdot\right)$. Then we consider the vector-valued sequence spaces $\ell_{q}\left(2^{-\theta m} G_{m}\right)$ and $\ell_{q}\left(2^{-\theta m} F_{m}\right)$. The relationship between the first space and $\bar{A}_{\theta, q ; J}$ is given by the metric surjection $\pi\left\{u_{m}\right\}=\sum_{m \in \mathbb{Z}} u_{m}$. The connection between $\bar{B}_{\theta, q ; K}$ and $\ell_{q}\left(2^{-\theta m} F_{m}\right)$ is given by the isometric embedding $\iota b=(\ldots, b, b, b, \ldots)$. The relevant diagram to keep in mind is

$$
\begin{array}{ccccccc}
\ell_{1}\left(G_{m}\right) & \stackrel{\pi}{\rightarrow} & A_{0} & \stackrel{T}{\rightarrow} & B_{0} & \stackrel{\iota}{\rightarrow} & \ell_{\infty}\left(F_{m}\right) \\
\ell_{1}\left(2^{-m} G_{m}\right) & \stackrel{\pi}{\rightarrow} & A_{1} & \stackrel{\rightarrow}{\rightarrow} & B_{1} & \stackrel{\iota}{\rightarrow} & \ell_{\infty}\left(2^{-m} F_{m}\right)
\end{array} .
$$

Besides $\pi$ and $\iota$ the following sequences of operators will be useful for establishing (1.2):

$P_{n}\left\{u_{m}\right\}=\left\{\ldots 0, u_{-n}, \ldots, u_{n}, 0, \ldots\right\}, Q_{n}^{+}\left\{u_{m}\right\}=\left\{\ldots 0,0, u_{n+1}, u_{n+2}, \ldots\right\}$, and $Q_{n}^{-}=I-P_{n}-Q_{n}^{+}$. They satisfy

$$
\left\|Q_{n}^{+}\right\|_{\ell_{1}\left(G_{m}\right), \ell_{1}\left(2^{-m} G_{m}\right)}=2^{-(n+1)}=\left\|Q_{n}^{-}\right\|_{\ell_{1}\left(2^{-m} G_{m}\right), \ell_{1}\left(G_{m}\right)}
$$

and

$$
\left\|P_{n}\right\|_{\ell_{1}\left(G_{m}\right), \ell_{1}\left(2^{-m} G_{m}\right)}=2^{n}=\left\|P_{n}\right\|_{\ell_{1}\left(2^{-m} G_{m}\right), \ell_{1}\left(G_{m}\right)} .
$$

Similar equalities hold when these operators are considered acting on the couple of $\ell_{\infty}$-spaces modelled on $\left\{F_{m}\right\}$.

Since $\pi$ is a metric surjection, we have

$$
\beta\left(T_{\bar{A}_{\theta, q ;}, \bar{B}_{\theta, q ; K}}\right)=\beta\left(T \pi_{\ell_{q}\left(2^{-\theta m} G_{m}\right), \bar{B}_{\theta, q ; K}}\right) \leq \beta\left(T \pi P_{2 n}\right)+\beta\left(T \pi\left(Q_{2 n}^{+}+Q_{2 n}^{-}\right)\right) .
$$


To deal with the first term, we use a functional which is equivalent to $\beta$. Given $S \in \mathcal{L}(A, B)$, let $\gamma(S)$ be the infimum of the set of all $\sigma>0$ for which there is a Banach space $Z$ and a compact linear operator $R \in \mathcal{L}(A, Z)$ such that

$$
\|S x\|_{B} \leq \sigma\|x\|_{A}+\|R x\|_{Z} \quad \text { for all } \quad x \in A .
$$

It turns out that $\frac{1}{2} \beta(S) \leq \gamma(S) \leq 2 \beta(S)$. This equivalence allows us to establish that

$$
\beta\left(T \pi P_{2 n}\right) \leq C \beta\left(T_{A_{0}, B_{0}}\right)^{1-\theta} \beta\left(T_{A_{1}, B_{1}}\right)^{\theta} \quad \text { for all } n \in \mathbb{N} .
$$

For the other terms, we have

$$
\begin{aligned}
\beta\left(T \pi\left(Q_{2 n}^{+}+Q_{2 n}^{-}\right)\right) \leq & 2 \beta\left(\left(P_{n}+Q_{n}^{+}+Q_{n}^{-}\right) \iota T \pi\left(Q_{2 n}^{+}+Q_{2 n}^{-}\right)\right) \\
\leq & 2\left[\beta\left(P_{n} \iota T \pi Q_{2 n}^{+}\right)+\beta\left(P_{n} \iota T \pi Q_{2 n}^{-}\right)\right. \\
& +\beta\left(Q_{n}^{+} \iota T \pi Q_{2 n}^{-}\right)+\beta\left(Q_{n}^{-} \iota T \pi Q_{2 n}^{+}\right) \\
& \left.+\beta\left(Q_{n}^{+} \iota T \pi Q_{2 n}^{+}\right)+\beta\left(Q_{n}^{-} \iota T \pi Q_{2 n}^{-}\right)\right],
\end{aligned}
$$

where the last six operators act from $\ell_{q}\left(2^{-\theta m} G_{m}\right)$ into $\ell_{q}\left(2^{-\theta m} F_{m}\right)$. We estimate each of these terms by the norm of the corresponding operator. Using properties of $P_{n}, Q_{n}^{+}, Q_{n}^{-}$, it follows that given any $\varepsilon>0$, there is $N \in \mathbb{N}$ such that

$$
\beta\left(T \pi\left(Q_{2 n}^{+}+Q_{2 n}^{-}\right)\right) \leq(1+\varepsilon) C \beta\left(T_{A_{0}, B_{0}}\right)^{1-\theta} \beta\left(T_{A_{1}, B_{1}}\right)^{\theta} \quad \text { for any } n \geq N .
$$

Full details can be found in $\left[{ }^{12}\right]$. It is also shown there that this approach works to estimate the measure of noncompactness of operators interpolated by the general real method $\left(A_{0}, A_{1}\right)_{\Gamma}$. The spaces $\left(A_{0}, A_{1}\right)_{\Gamma}$ are defined by replacing the weighted $\ell_{q}$-norm of $\left(A_{0}, A_{1}\right)_{\theta, q}$ by a more general lattice norm (see $\left.\left[{ }^{13}\right]\right)$.

\section{ACKNOWLEDGEMENTS}

This research was supported in part by the Ministry of Education and Science of Spain (project MTM2004-01888) and CAM-UCM (Research group 910348). F. Cobos was partly supported by RTM PHD FP6-511953.

\section{REFERENCES}

1. Bergh, J. and Löfström, J. Interpolation Spaces. An Introduction. Springer, Berlin, 1976.

2. Triebel, H. Interpolation Theory, Function Spaces, Differential Operators. NorthHolland, Amsterdam, 1978.

3. Cobos, F., Manzano, A. and Martínez, A. Interpolation theory and measures related to operator ideals. Quart. J. Math., 1999, 50, 401-416.

4. Cobos, F. and Martínez, A. Remarks on interpolation properties of the measure of weak non-compactness and ideal variations. Math. Nachr., 1999, 208, 93-100. 
5. Cobos, F., Fernández-Martínez, P. and Martínez, A. Interpolation of the measure of noncompactness by the real method. Studia Math., 1999, 135, 25-38.

6. Schechter, M. Principles of Functional Analysis. American Mathematical Society, Providence, 2002.

7. Cobos, F., Edmunds, D. E. and Potter, A. J. B. Real interpolation and compact linear operators. J. Funct. Anal., 1990, 88, 397-401.

8. Cobos, F. and Fernandez, D. L. On interpolation of compact operators. Ark. Mat., 1989, 27, 211-217.

9. Cobos, F. and Peetre, J. Interpolation of compactness using Aronszajn-Gagliardo functors. Israel J. Math., 1989, 68, 220-240.

10. Cobos, F., Kühn, T. and Schonbek, T. One-sided compactness results for AronszajnGagliardo functors. J. Funct. Anal., 1992, 106, 274-313.

11. Teixeira, M. F. and Edmunds, D. E. Interpolation theory and measure of non-compactness. Math. Nachr., 1981, 104, 129-135.

12. Cobos, F., Fernández-Cabrera, L. M. and Martínez, A. Abstract $K$ and $J$ spaces and measure of non-compactness. Math. Nachr. (to appear).

13. Nilsson, P. Reiteration theorems for real interpolation and approximation spaces. Ann. Mat. Pura Appl., 1982, 132, 291-330.

\section{Mittekompaktsuse mõõdu interpoleerimine}

\section{Fernando Cobos, Luz M. Fernández-Cabrera ja Antón Martínez}

On revideeritud tuntud tulemusi mittekompaktsuse mõõdu interpoleerimise kohta. On esitatud uus lähenemine interpolatsiooni valemi tõestamiseks reaalse meetodi puhul. 